\title{
Reexpansion Pulmonary Edema
}

\author{
Partha Pratim Chakraborty • Swarup Chakraborty
}

Received: 22 January 2009/Accepted: 27 April 2009/Published online: 8 April 2011

(C) Association of Surgeons of India 2011

\section{Introduction}

Reexpansion pulmonary edema (RPE) is a relatively rare condition which develops when a collapsed lung is allowed to expand suddenly. Histological abnormalities of the pulmonary micro-vessels as well as mechanical stress exerted during reexpansion are implicated in the pathogenesis of this disorder. A good outcome can be expected from symptomatic therapy and procedures that reduce the level of mechanical stress during reexpansion.

\section{Case Report}

A 31 year old male presented to the emergency with respiratory distress and persistent cough for last 4 days. These complaints were preceded by right sided chest discomfort. On admission his blood pressure was 128/ $74 \mathrm{~mm}$ of $\mathrm{Hg}$ with pulse rate of $104 / \mathrm{mn}$ and respiratory rate of $34 / \mathrm{mn}$. The $\mathrm{SpO} 2$ was $86 \%$ on room air which went up to $92 \%$ with supplemental oxygen (6lt/mn by nasal prongs). Systemic examination revealed absent breath sounds over the right chest, which was tympanitic on percussion. The mediastinum was shifted to left. Rest of the systemic examination was non-contributory. The chest X-ray was consistent with the clinical diagnosis of right sided pneumothorax (Fig. 1a). Baseline investigations were within normal limits.

$\mathrm{He}$ underwent intercostal tube drainage under local anesthesia in the fourth intercostal space behind the right

P. P. Chakraborty $(\bowtie) \cdot$ S. Chakraborty

Midnapore Medical College,

Midnapore, West Bengal, India

e-mail: docparthapc@yahoo.co.in anterior axillary line. Drainage was started without negative pressure suction with a $16 \mathrm{Fr}$ chest tube. About an hour following the procedure the patient developed severe dyspnoea with violent cough and production of pinkish sputum. The pulse was $164 / \mathrm{mn}$ and the blood pressure was $64 / 48 \mathrm{~mm}$ of $\mathrm{Hg}$. The $\mathrm{SpO} 2$ went down to $64 \%$. The right hemithorax was full of crepitations, left being absolutely clear. Chest x-ray documented fluffy opacities scattered along the whole right lung field (Fig. 1b).

The patient received supplemental oxygen via a nonrebreathing face mask to compensate for hypoxemia. He was put on IV fluids, inotropes (Dopamine $5-15 \mathrm{mcg} / \mathrm{kg} /$ $\mathrm{mn}$ ) and antibiotics. Patient showed gradual clinical improvement and the inotrope support was withdrawn on 3rd post admission day. The vitals remained stable. The Intercostal tube was removed on 4th admission day as there was no air leak. The patient recovered completely without residual hypoxemia. Repeat $\mathrm{x}$-ray at that time showed minimal opacities over the right mid and lower zones (Fig. 2a). Conservative management was continued along with respiratory physiotherapy. The pre-discharge $\mathrm{x}$-ray done on 7th day was absolutely normal (Fig. 2b).

\section{Discussion}

When a collapsed lung reexpands after an extended duration (generally $>72 \mathrm{hrs}$ ), pulmonary edema sometimes occurs in it. This is called RPE [1]. RPE is a relatively uncommon condition which typically appears unexpectedly and rapidly. RPE is usually unilateral and occurs when the lung is rapidly re-expanded by active evacuation of large amounts of air or fluid from the pleural space. The etiopathogenesis of RPE is still obscure and is probably multifactorial making it difficult to suggest a precise 
Fig. 1 a Chest X-ray showing hyperlucency of right hemithorax, absent broncho-vascular markings and contralateral mediastinal shift consistent with right sided pneumothorax.

b Chest x-ray documenting fluffy opacities scattered along the whole right lung field
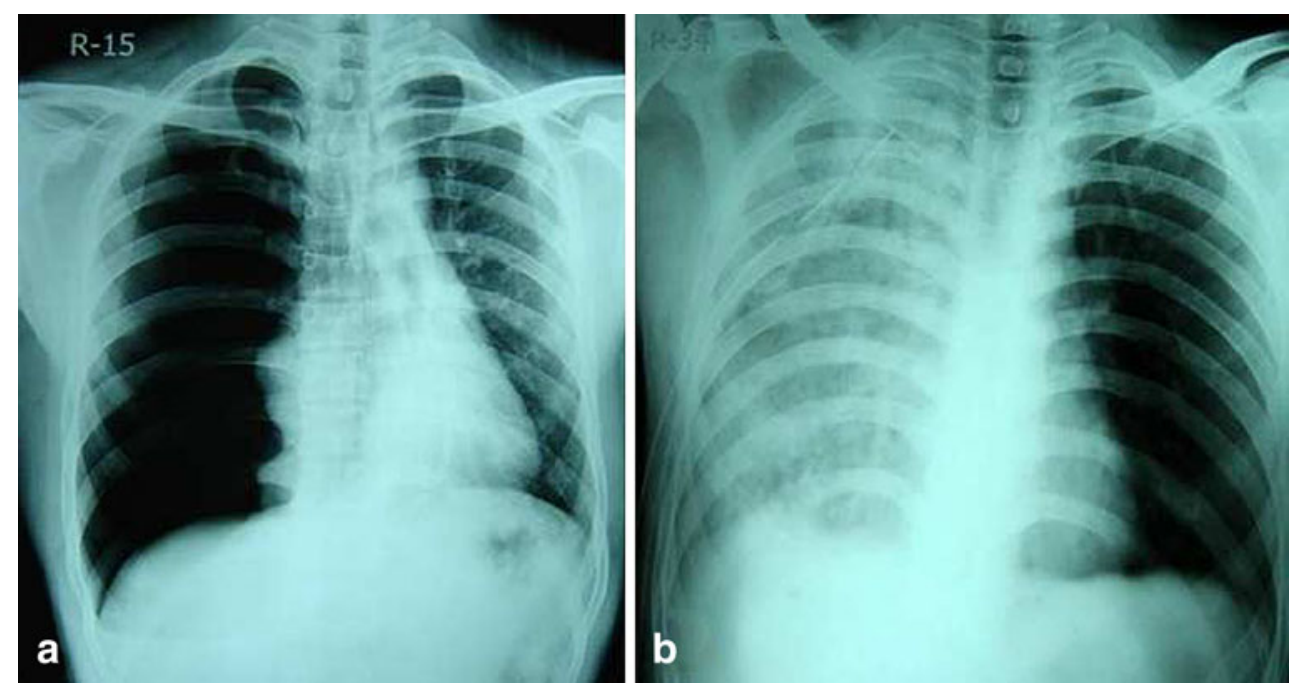

therapeutic measure. Implicated in the etiological process of RPE are mechanical features like duration and severity of collapse, technique of re-expansion, increased pulmonary vascular permeability, airway obstruction, pulmonary artery pressure changes or inflammatory changes (production of Inerleukin 8, Leukotrienne B4 and loss of surfactant) within the pulmonary vasculature.

RPE is a rare and potentially lethal complication with 15 to $20 \%$ mortality [2]. Supportive therapy is the rule once this condition sets in. To avoid this dreaded complication the risk factors for RPE should be evaluated and considered prior to the insertion of chest tubes. The following risk factors have been associated with RPE: younger age, longer duration of a lung a quick lung reexpansion. Nevertheless, it has been seen that RPE can develop even when the pulmonary collapse is of short duration and the lung is reexpanded without suction as in this particular patient. In patients with the risk factors, the thoracostomy tube should be initially left off suction in an effort to prevent RPE primarily.

Mild cases of RPE may be reversed with conventional ventilator therapy and medications. A case report has shown that drugs like sivelestat sodium hydrate may sometimes be useful [3]. Asynchronous differential lung ventilation is now being considered as a relatively new therapeutic modality for RPE [4].

To conclude, pulmonary edema should always be considered if the patient's respiratory condition worsens after lung reexpansion and emergency surgeons treating chronic lung collapse must be aware of this possibility and try to prevent the occurrence of this complication, which is far from being benign. In the future, it is expected that the cause of histological changes of the pulmonary microvessels in a chronically collapsed lung will be revealed, and appropriate therapies will therefore be developed according to this cause.
Fig. 2 a Repeat x-ray on 4th day showing minimal opacities over the right mid and lower zones. b Normal chest $\mathrm{x}$-ray before discharge
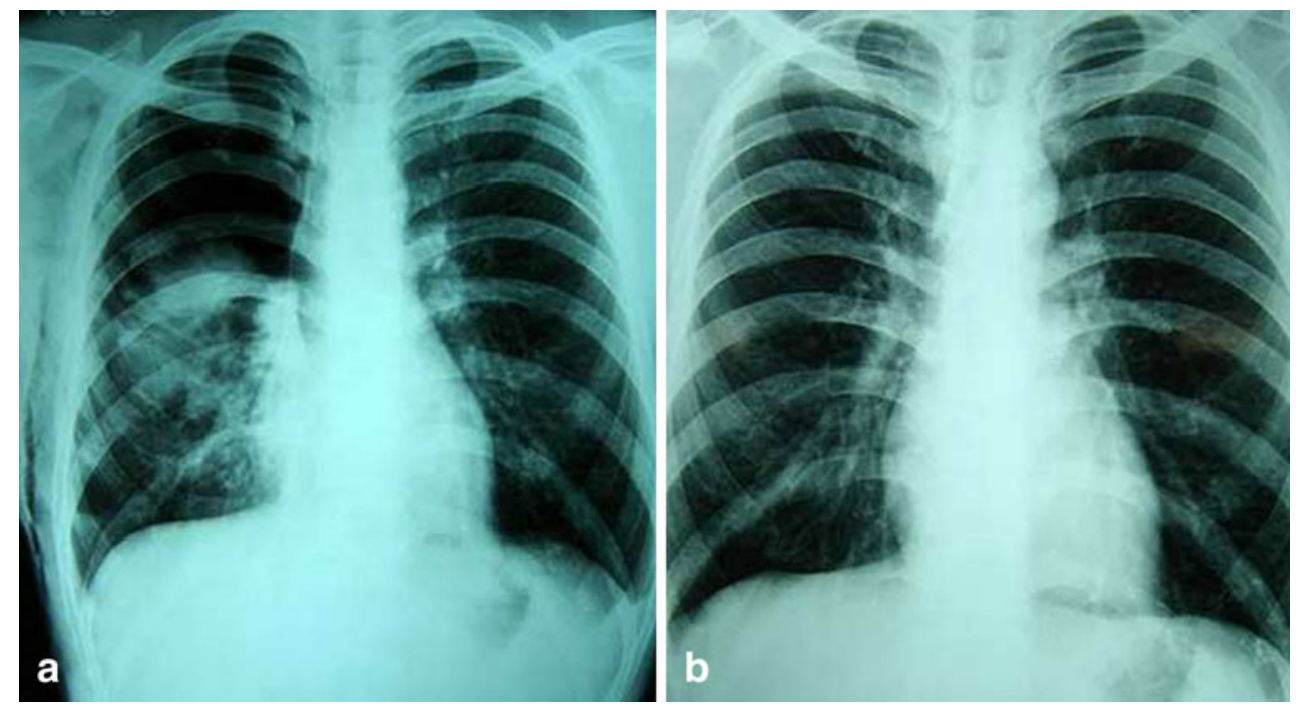


\section{References}

1. Sohara Y (2008) Reexpansion pulmonary edema. Ann Thorac Cardiovasc Surg 14:205-209

2. Sherman SC (2003) Reexpansion pulmonary edema: a case report and review of the current literature. J Emerg Med 24:23-27
3. Kimura Y, Endo K, Tsukahara A, Shimoyama M, Mitsui M, Takano K, Hayashi K (2005) Clinical experience of sivelestat sodium hydrate for severe reexpansion pulmonary edema; report of a case. Kyobu Geka 58:1010-1012

4. Cho SR, Lee JS, Kim MS (2005) New treatment method for reexpansion pulmonary edema: differential lung ventilation. Ann Thorac Surg 80:1933-1934 